\title{
Direct observation of domain wall motion and lattice strain dynamics in ferroelectrics under high-power resonance
}

\author{
Mihail Slabki $\odot,{ }^{1, *}$ Lalitha Kodumudi Venkataraman $\odot,{ }^{1}$ Stefano Checchia $\odot,{ }^{2}$ Lovro Fulanović $\odot,{ }^{1}$ \\ John Daniels ${ }^{\circ},{ }^{3}$ and Jurij Koruza $\mathbb{1}^{1, \dagger}$ \\ ${ }^{1}$ Department of Materials and Earth Sciences, Technical University of Darmstadt, Alarich-Weiss-Straße 2, Darmstadt, Germany \\ ${ }^{2}$ European Synchrotron Radiation Facility (ESRF), 71 Avenue des Martyrs, Grenoble, France \\ ${ }^{3}$ School of Materials Science and Engineering, University of New South Wales, New South Wales 2052, Sydney, Australia
}

(Received 22 November 2020; revised 6 April 2021; accepted 4 May 2021; published 26 May 2021)

\begin{abstract}
Domain wall motion and lattice strain dynamics of ferroelectrics at resonance were simultaneously measured by combining high-power burst excitation and in situ high-energy x-ray diffraction. The increased loss at high vibration velocity was directly related to the increased domain wall motion, driven by dynamic mechanical stress. A general relationship between the microstructural strain contributions and macroscopic electromechanical behavior was established, allowing the prediction of high-power stability of ferroelectric materials. The results indicate that the materials' stability during high-power drive is predominantly related to the basic chemical composition, while the piezoelectric hardening mechanisms mainly influence the small-signal behavior.
\end{abstract}

DOI: 10.1103/PhysRevB.103.174113

\section{INTRODUCTION}

Ferroelectrics exhibit strong electromechanical coupling, originating from a combination of the inherent anisotropy of their crystal lattice (intrinsic contribution) and the presence of ferroelectric domain walls (extrinsic contribution) ${ }^{1}$ [1]. External perturbations, such as electric field $E$ or mechanical stress $T$, influence the system by favoring one of the polarization directions and exerting a force on domain walls, initiating their vibration and/or movement [2]. The domain wall motion contributes significantly to the electromechanical response, but is also the origin of hysteretic behavior and determines the loss in these materials [3,4]. Understanding the underlying physics of domain walls is thus crucial for their functionalization and development of new devices $[5,6]$.

Domain walls are interfaces separating regions with uniform direction and amplitude of the spontaneous polarization and strain, which are pinned by a defect-generated variation in potential. Their dynamics have been studied through macroscopic measurements [3,7] and in recent years, direct evidence for their role in property variations has been provided by diffraction methods [8]. The intrinsic $h k l$-dependent lattice strain and extrinsic domain switching strain have been investigated in situ under the application of an electric field, without changing the material's natural boundary conditions. Strain contributions under quasistatic electric field [9-11] or dynamic low-frequency (Hz-range) subcoercive loading [12-15] have been deconvoluted. However, the motion of ferroelectric domain walls at resonance excitation has not been investigated yet.

\footnotetext{
*Corresponding author: slabki@ ceramics.tu-darmstadt.de

†Corresponding author: koruza@ ceramics.tu-darmstadt.de

${ }^{1}$ Other extrinsic contributions, e.g., field-induced phase transformations, are also possible, but have not been observed in this study.
}

In piezoelectrics, or generally in electromechanically active materials (e.g., poled polycrystalline ferroelectrics), oscillating vibration strain in $j$ direction $\varepsilon_{j \text {,vibro }}$ can be induced through the electromechanical $\varepsilon_{j, \text { el }}$ and mechanical $\varepsilon_{j \text {,mech }}$ coupling by applying an alternating electric field or mechanical stress, respectively. The strain generation is described by the constitutive equation

$$
\varepsilon_{j, \text { vibro }}=\varepsilon_{j, \mathrm{el}}+\varepsilon_{j, \mathrm{mech}}=d_{i j} E_{i}+s_{i j}^{E} T_{i},
$$

where $d_{i j}$ is the piezoelectric coefficient and $s_{i j}^{E}$ is the elastic compliance, while $\varepsilon_{j, \text { vibro }}=2 u / l$ is determined by the edge displacement $u$ and the sample length $l$. Moreover, exciting the material at the piezoelectric resonance, also denoted as self-resonance, offers an additional unique opportunity to induce large strain amplitude at relatively low applied electric fields. This driving strategy is being widely used in ultrasonic transducers and motors [16,17]. These applications are usually referred to as high-power electromechanical converters, where the term "high-power drive" denotes the excitation of the piezoelement at its first- or higher-order resonance frequency [18]. A piezoelectric driven at or in the vicinity of its resonance frequency $f^{R}$ will exhibit a large increase in strain, proportional to $\left(8 Q_{i j}^{R}\right) / \pi^{2}$ [19]. Here, $Q_{i j}^{R}$ is the resonance quality factor, which is inversely proportional to the loss in the material during vibration and is thus one of the most important piezoelectric coefficients. Note that $Q_{i j}^{R}$ directly indicates the enhancement of the sample's vibration velocity $v$ since

$$
v_{j}=\pi f^{R} l \varepsilon_{j, \text { vibro }},
$$

where $l$ is the length in the vibration direction (modified after [20]). However, in ferroelectrics, the $Q_{i j}^{R}$ usually exhibits strong nonlinear behavior when the amplitude of the applied electric field increases. Therefore, the applied electric field 
and the induced vibration velocity exhibit a nonlinear relation and the increase of vibration velocity is limited due to a simultaneous drop of $Q_{i j}^{R}$, accompanied by strong self-heating of the sample and eventual depolarization [20,21]. Prior research, based on indirect electrical measurements, related this observation to enhanced non- $180^{\circ}$ domain wall motion [21-23]. Nevertheless, the exact driving force for this phenomenon is unclear and direct structural evidence is missing. Note that the applied electric field amplitudes in the resonance are comparably low, i.e., well below the pinning-depinning transition [24], thus the electric field alone cannot be considered as the driving force for non- $180^{\circ}$ domain wall motion. Some studies have therefore speculated that the large strain and the domain wall motion at resonance is induced by the dynamic (tensile and compressive) mechanical stress [22,25].

This work experimentally investigates the microscopic origins of the large electromechanical strain response at and near the piezoelectric resonance and at high vibration velocity. To provide insight into the mechanisms of domain wall motion and lattice strain during resonance drive, we combined stroboscopic time-resolved in situ high-energy x-ray diffraction with piezoelectric pulse drive measurement and observed the macroscopic and microscopic strain simultaneously in real time. In particular, we examined the origin of the $Q_{i j}^{R}$ decrease and established evidence for the induced mechanical stress being the dominant driving force for domain wall motion. Ferroelectrically hard and soft compositions of $\mathrm{Pb}(\mathrm{Zr}, \mathrm{Ti}) \mathrm{O}_{3}$ (polycrystalline bulk PZT; PIC-151 and -181, PI Ceramic $\mathrm{GmbH}$, Germany) were compared to reveal the influence of defect-based inhibition and enhancement of domain wall motion on the loss mechanism.

\section{EXPERIMENT}

The samples $\left(24 \times 1 \times 1 \mathrm{~mm}^{3}\right)$ were poled at $120^{\circ} \mathrm{C}$ for 10 min with $2 \mathrm{kV} / \mathrm{mm}$ (PIC-151) and $3 \mathrm{kV} / \mathrm{mm}$ (PIC-181). Poled samples were excited in the transverse length vibration mode (31 mode) [26] with an AC sinusoidal electric field applied in the 3 direction, $E_{3}$, with a frequency at or near their piezoelectric resonance using a high-power experimental setup with the pulse drive method and burst excitation (Fig. 1). The samples were driven by a pulse with $5000-10000$ cycles and the electromechanical coefficients were derived from the transient vibration decay after short-circuiting the sample, as described elsewhere [27,28]. The low number of driving cycles prevented the sample's self-heating. The first set of experiments [Figs. 2(a) and 2(b)] was carried out at different electric field amplitudes and the corresponding resonance frequencies to achieve the desired vibration velocity [according to Eq. (1) in [28]; Table S1 of the Supplemental Material [29]]; the second set was carried out as a sweep across different frequencies using a constant electric field amplitude [Figs. 2(c) and 2(d)].

The diffraction patterns were collected in situ during sample excitation at the ID15 beamline [30] of the European Synchrotron Radiation Facility (ESRF) and the PETRA III P02.1 beamline of the Deutsches Elektronen-Synchrotron (DESY) in transmission geometry. The $0.3 \times 0.3 \mathrm{~mm}^{2} \mathrm{x}$-ray beam with an energy of 75 and $60 \mathrm{keV}$, respectively, was positioned in the center of the vibrating sample. Diffraction

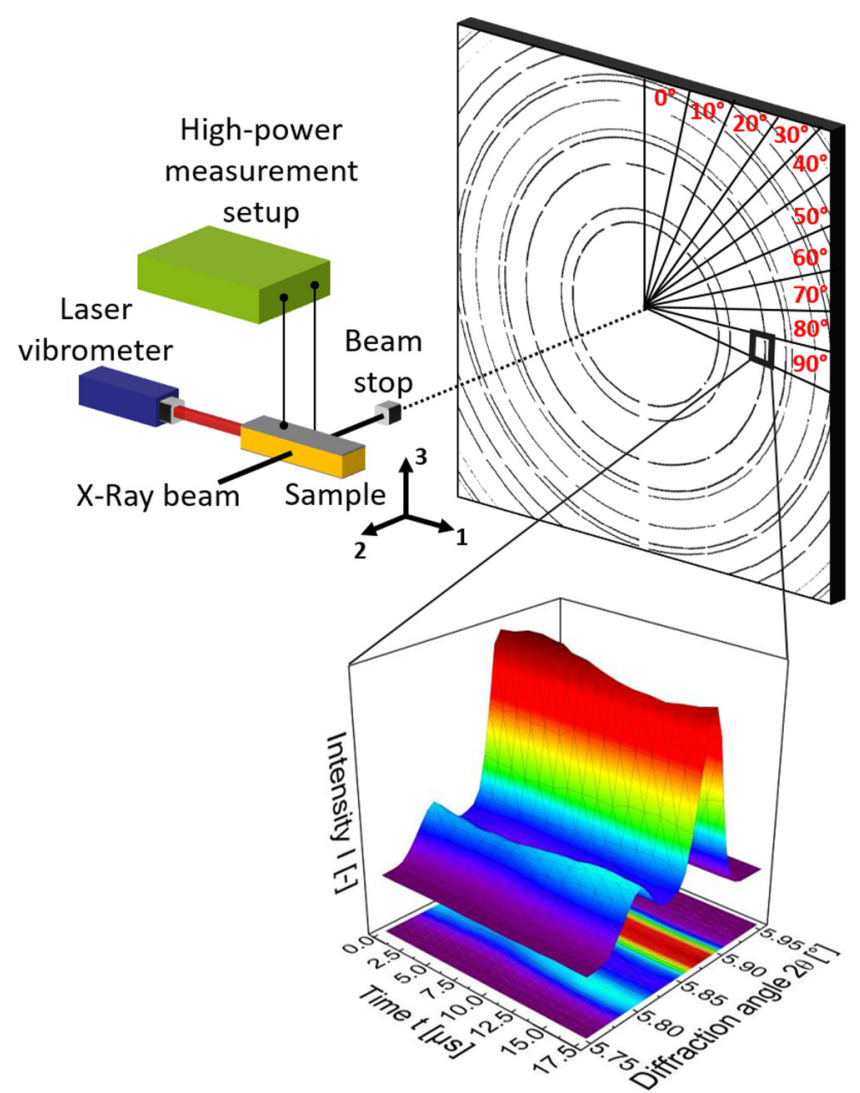

FIG. 1. Schematics of the high-power piezoelectric pulse drive measurement with a laser vibrometer combined with a time-resolved in situ synchrotron diffraction measurement. Electric field was applied in the 3 direction $\left(E_{3}\right)$, while largest strain was realized in the 1 direction $\left(\varepsilon_{1}\right)$ due to the fundamental transverse resonance vibration mode (31). The inset shows exemplarily a reconstructed $2 \theta$ section of a diffractogram between $5.75^{\circ}$ and $5.95^{\circ}$ including the $(002 / 200)$ doublet reflection with the scattering vector in the 1 direction perpendicular to the applied electric field. The time scale depicts the intensity evolution over the entire sinusoidal field cycle. The intensity interchange between the two reflections during the positive and negative field states directly evidences the motion of non- $180^{\circ}$ domain walls during the soft PZT sample's resonance vibration at $1.5 \mathrm{~m} / \mathrm{s}$.

patterns were collected using a Pilatus CdTe 2M area detector (Dectris Ltd., Switzerland). Time-resolved data collection with stroboscopic acquisition [15,31] was used and each highfrequency sinusoidal cycle was described by 16 segments, whereby summation of the cycles ensured sufficient intensities of the diffracted rings.

The Debye-Scherer rings were divided into $10^{\circ}$ sections over the azimuthal angle $\alpha$ (Fig. 1). The pattern within each section was integrated to obtain one-dimensional diffractograms with the diffracted intensities as a function of the diffraction angle $2 \theta$ from $2^{\circ}$ to $9^{\circ}$ [32]. The $h k l$-diffraction peaks and double peaks (111), (002/200), (022/220), and (211/112) were fitted by single and double Gaussian functions, respectively. The orientation-dependent $h k l$ lattice strain $\varepsilon_{h k l}$ and the domain switching fraction $\eta_{002}$ were determined from the change in lattice spacing and the intensity interchange [33] (see the Supplemental Material [29]). The total 

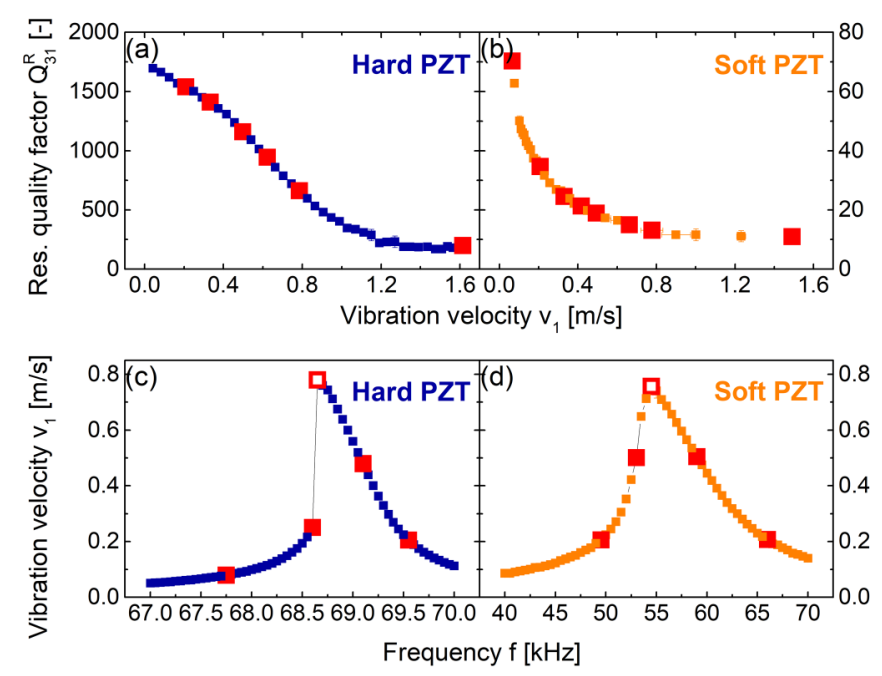

FIG. 2. (a), (b) Experimentally determined resonance quality factor in transverse length mode $\left(Q_{31}^{R}\right)$ as a function of macroscopic vibration velocity $\left(v_{1}\right)$ measured at the sample's edge. The maximum electric field amplitudes $\left(E_{3}^{\max }\right)$ were $40 \mathrm{~V} / \mathrm{mm}$ (hard PZT) and $120 \mathrm{~V} / \mathrm{mm}$ (soft PZT). (c), (d) Vibration velocity $\left(v_{1}\right)$ as a function of the driving frequency $(f)$ at and around the samples' resonance frequency ( $f^{R}$, indicated by open symbols) at constant electric field amplitudes $\left(E_{3}\right)$ of $6.4 \mathrm{~V} / \mathrm{mm}$ (hard PZT) and $74 \mathrm{~V} / \mathrm{mm}$ (soft PZT). Measurements were done with decreasing frequency. Red points mark the conditions at which the diffraction experiments were conducted.

lattice strain $\varepsilon_{\text {lattice }}$ was calculated by a weighted sum of the seven crystallographic planes strains using the Voigt approximation [34], utilizing the texture and multiplicity factors of individual planes (Table I). The strain contribution from non- $180^{\circ}$ domain wall motion, $\varepsilon_{\text {non- }-180^{\circ}}$, was calculated using a volume-weighted average of the lattice distortion of the domains over the entire orientation space [33,35], where the domain switching fraction was determined from the intensity interchange of the (002/200) diffraction peaks (Fig. 1). $\alpha=0^{\circ}$ was chosen to be parallel to the direction of the

TABLE I. Overview of the utilized strain coefficients $\left(\varepsilon_{j}\right)$, grouped by their determination. The diffraction analysis was carried out locally in the sample center, strains from the pulse drive measurements are sample average taken at the edge, while the FEM simulated values were calculated at the sample center and as sample average for comparison.

Strains measured by diffraction

$\varepsilon_{j \text {,lattice }} \quad$ Piezoelectric lattice strain

$\varepsilon_{j, \text { non-180 }} \quad$ Strain contribution from non- $180^{\circ}$ domain wall motion

$\varepsilon_{j, \mathrm{XRD}} \quad$ Total sample strain determined from XRD

Strains measured by laser vibrometer

$\varepsilon_{j, \mathrm{el}} \quad$ Electromechanically induced strain

$\varepsilon_{j, \text { mech }} \quad$ Mechanically induced strain

$\varepsilon_{j, \text { vibro }} \quad$ Total vibration strain

Strain calculated by FEM simulation

$\varepsilon_{j, \mathrm{FEM}} \quad$ FEM simulated strain electric field $\left(E_{3}\right)$ for the strain calculation in the longitudinal 3 direction $\left(\varepsilon_{3, \text { non-180 }}\right)$ and perpendicular to the field direction for the strain calculation in the transverse 1 direction $\left(\varepsilon_{1, \text { non-180 }}\right)$ (Fig. 1). A finite element method (FEM) model of the sample vibrating at a maximum velocity of 0.6 $\mathrm{m} / \mathrm{s}$ was created (COMSOL Multiphysics Inc., USA), whereby a $0.01-\mathrm{mm}$ mesh size was implemented to determine the strain and stress distributions in the sample during resonance vibration.

\section{RESULTS}

\section{A. Quality factor decrease and domain wall motion}

The high-power resonance quality factors in transverse length mode $Q_{31}^{R}$ are shown in Figs. 2(a) and 2(b). Hard PZT reveals large $Q_{31}^{R}$ values at small vibration velocity, which is attributed to $\mathrm{Mn}$ acceptor doping and the subsequent formation of charge-compensating oxygen vacancies. These defects stabilize the domain structure and inhibit domain wall motion, resulting in lower loss [36-38]. $Q_{31}^{R}$ values of soft PZT are significantly lower due to enhanced domain wall mobility induced by $\mathrm{Sb}$ donor doping and $A$-site vacancy formation. In both materials $Q_{31}^{R}$ strongly decreases at large vibration velocity $[23,39,40]$. This was predicted to be related to enhanced motion of ferroelectric/ferroelastic domain walls [21], which is to be proven in the following by structural evidence in terms of microstructural strain contributions.

In order to analyze the correlation between decreasing $Q_{31}^{R}$ values and domain wall motion in resonance, we selected several representative vibration velocities [red symbols in Figs. 2(a) and 2(b)] and determined the different contributions to the total sample strain during these high-power drive conditions in situ using synchrotron radiation (Table I). The measured transverse strains $\varepsilon_{1}\left(\perp E_{3}\right)$ are shown in Fig. 3 and the longitudinal strains $\varepsilon_{3}\left(\| E_{3}\right)$ in Fig. S1 [29]. The decrease of $Q_{31}^{R}$ with increasing vibration velocity is accompanied by an increase of $\varepsilon_{\text {lattice }}$ and $\varepsilon_{\text {non }-180^{\circ}}$. Both strain contributions are largest in the 1 direction, confirming the transverse (31) vibration as the largest-amplitude mode. While the $\varepsilon_{\text {lattice }}$ values are comparable in both materials, the $\varepsilon_{\text {non }-180^{\circ}}$ is lower in hard PZT, which is a result of ferroelectric hardening. The stabilization of the domain structure is also evident by a relatively small increase of $\varepsilon_{\text {non }-180^{\circ}}$ in hard PZT with increasing vibration velocity, which is larger in soft PZT.

The contribution of $\varepsilon_{1, \text { non-180 }}$ to $\varepsilon_{1, \mathrm{XRD}}$ is depicted in Fig. 4. $\varepsilon_{1, \mathrm{XRD}}$ is the total sample strain determined from the diffraction analysis and the sum of the two contributions $\varepsilon_{1, \text { lattice }}$ and $\varepsilon_{1, \text { non- }-180^{\circ}}$ [41], since no other extrinsic strain contribution was detected. The $\varepsilon_{1, \mathrm{non}-180^{\circ}} / \varepsilon_{1, \mathrm{XRD}}$ ratio clearly increases at large vibration velocity, which is a direct evidence for the correlation between enhanced domain wall motion and decrease of $Q_{31}^{R}$. This contribution is significantly larger in soft PZT, which explains the much lower $Q_{31}^{R}$ of this material. Interestingly, the domain wall contribution (7-15\% for hard and $12-35 \%$ for soft PZT) is in general slightly lower as compared to off-resonance conditions at lower frequencies $[8,12]$, which might be the consequence of the frequency-dependent domain wall response $[4,42,43]$. We note that although higher 


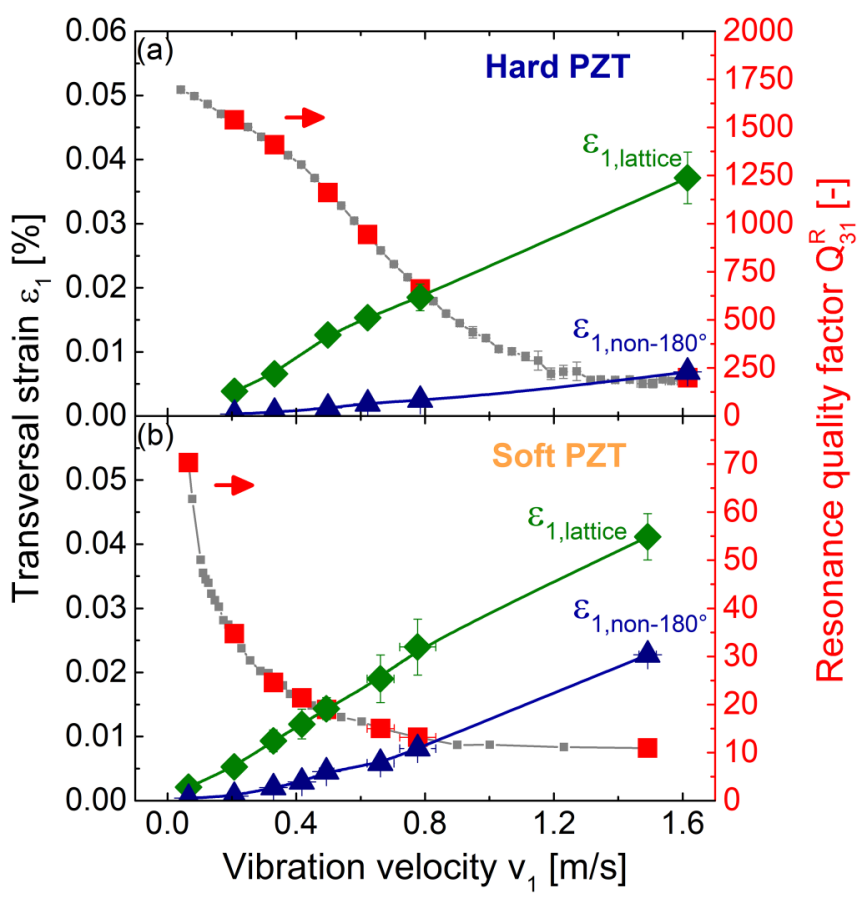

FIG. 3. Lattice strain $\left(\varepsilon_{1, \text { lattice }}\right)$ and strain contribution from non$180^{\circ}$ domain wall motion $\left(\varepsilon_{1, \text { non- } 180^{\circ}}\right)$, determined from the XRD measurement, in comparison to the resonance quality factor $\left(Q_{31}^{R}\right)$, determined from the pulse drive measurement, as a function of edge vibration velocity $\left(v_{1}\right)$ perpendicular to the applied electric field $\left(E_{3}\right.$, 31 vibration mode) for (a) hard and (b) soft PZT. The same analysis for the 33 vibration mode (longitudinal strain $\varepsilon_{3}$ ) is shown in Fig. S1 [29].

electric field amplitudes were used for soft PZT to achieve the same vibration velocity (soft PZT: $E_{3}^{\max }=120 \mathrm{~V} / \mathrm{mm}$, hard PZT: $E_{3}^{\max }=40 \mathrm{~V} / \mathrm{mm}$ ), this cannot explain the observed difference in the strain contributions.

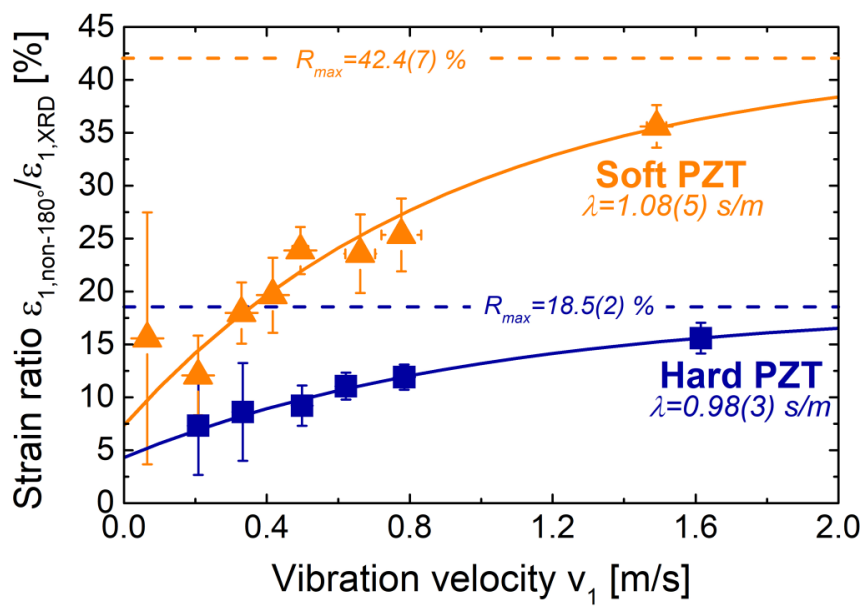

FIG. 4. Strain ratio revealing the relative strain contribution from non- $180^{\circ}$ domain wall motion $\left(\varepsilon_{1, \text { non- }-180^{\circ}}\right)$ to the total strain determined by XRD measurement ( $\varepsilon_{1, \mathrm{XRD}}$, sum of $\varepsilon_{1 \text {,lattice }}$ and $\left.\varepsilon_{1, \text { non }-180^{\circ}}\right)$. Continuous lines depict the exponential dependency [Eq. (3)], while dotted lines indicate the determined saturation ratio.

\section{B. Generalized relation between domain wall motion and quality factor}

Compositional modifications (acceptor/donor doping) change the material's $Q_{i j}^{R}$. However, they do not change the high-power behavior, i.e., the vibration velocity dependence of $Q_{i j}^{R}$, which is determined by the properties of the basic material. For example, small-signal $Q_{i j}^{R}$ variations by orders of magnitude were reported for doped PZT $[21,23,44]$ and other ferroelectrics; however, when measured under largesignal conditions, the PZT-based materials suffer a strong $Q_{i j}^{R}$ decrease at comparatively low vibration velocity, while no significant change was observed for $\mathrm{Na}_{1 / 2} \mathrm{Bi}_{1 / 2} \mathrm{TiO}_{3}$-based materials $[45,46]$. These macroscopic observations can be related to the microstructural findings in this study (Fig. 4). The strain ratios of hard and soft PZT increase and saturate at large vibration velocity (Fig. 4), which correlates with the decrease and stabilization of the $Q_{31}^{R}$ values (Fig. 3). These results provide a direct link between the microstructural parameters and the macroscopic behavior.

A similar increase and saturation of the strain contribution from domain wall motion were observed before under weakfield off-resonance conditions by macroscopic mechanical stress dependent [3] and microscopic electric field dependent [8] measurements. This behavior was described by the Rayleigh law and the motion of domain walls in an energy potential landscape [47]. It is assumed that the material behavior determined here (Fig. 4) is phenomenologically very similar; however, the formalism cannot be directly transferred, since the diffraction analysis does not allow a clear deconvolution of reversible and irreversible non- $180^{\circ}$ domain wall motion. Therefore, we suggest to describe the strain ratios (Fig. 4) by an associated exponential relation

$$
\frac{\varepsilon_{1, \mathrm{non}-180^{\circ}}}{\varepsilon_{1, \mathrm{XRD}}}=R_{\max }-R_{0} e^{-\lambda v_{1}} .
$$

$R_{\max }$ determines the maximum achievable strain ratio of the material. It depicts the strain contribution from non- $180^{\circ}$ domain wall motion at very large vibration velocity, yet below the ferroelectric switching region, i.e., within the so-called Rayleigh region [48]. The value is related to the stabilization of the domain configuration, e.g., by acceptor doping or other hardening mechanism. $R_{\max }-R_{0}$ determines the $y$-axis intercept at $0 \mathrm{~m} / \mathrm{s}$, i.e., the strain ratio at low vibration velocity. Other than in the Rayleigh formalism, the strain ratio does not decrease towards zero at zero vibration velocity, but approaches a finite value, since the strain contribution from all domain wall motion is considered, not only from the irreversible motion. Finally, $\lambda$ is the attenuation coefficient and describes the increase in the strain ratio. $\lambda$ is directly linked to the decrease of $Q_{31}^{R}$ as a function of vibration velocity. $R_{\max }-R_{0}$ is lower for hard PZT as compared to soft PZT (Table S2 [29]) and corresponds to the significantly larger small-signal $Q_{31}^{R} . R_{\max }$ is significantly lower in hard PZT as well, which is associated with the higher $Q_{31}^{R}$ values at large vibration velocity. Note that the absolute differences should be treated carefully due to the increasing experimental uncertainty at low vibration velocity and the limited number of data points at large vibration velocity. Interestingly, although the two PZT compositions reveal distinctly different $Q_{31}^{R}$ values, 
the $\lambda$ value is close to $1 \mathrm{~s} / \mathrm{m}$ for both materials (Fig. 4). This indicates a very similar $Q_{31}^{R}$ decrease with increasing vibration velocity, determined by the intrinsic properties of the basic tetragonal PZT, as pointed out above. It is therefore proposed that the attenuation coefficient could be considered as a characteristic parameter for a general evaluation of a ferroelectric material's resistance against a $Q_{31}^{R}$ decrease at large vibration velocity. It is expected that the high-power characteristic of material families can be generalized based on $\lambda$, which should be further experimentally confirmed in the future.

\section{Increasing domain wall motion in resonance}

Figures 2(c) and 2(d) depict the evolution of the vibration velocity during a frequency sweep across the samples' resonance frequency at a constant electric field amplitude. The field amplitude was selected such that a peak vibration velocity of $0.8 \mathrm{~m} / \mathrm{s}$ was reached at resonance. Peaks of both materials are asymmetric, which was previously also observed in frequency-dependent impedance measurements $[44,49,50]$ and is directly reflected in the vibration velocity measured here. The asymmetry is especially pronounced in hard PZT, exhibiting characteristic peak distortion around the resonance frequency, caused by the nonlinear vibration velocity dependence of the electromechanical coefficients. To evaluate the role of the electric field as the driving force for domain wall motion, $\varepsilon_{1 \text {,non- }-180^{\circ}}$ and $\varepsilon_{1 \text {,lattice }}$ were tracked in comparison to the vibration velocity at selected frequencies at and around the resonance frequency [red symbols in Figs. 2(c) and 2(d)]. The corresponding values are shown in Fig. 5. The $\varepsilon_{1 \text {, lattice but also }}$ the $\varepsilon_{1, \text { non- }-180^{\circ}}$ and thus the domain wall motion are clearly enhanced at the resonance, although the applied electric field amplitude is the same for all measurement points. This experimentally confirms that there must be another stimulus at the resonance (besides the externally applied electric field) that drives the domain wall motion.

\section{Origin of domain wall driving force in resonance}

To facilitate the determination of the domain wall driving forces, a correlation between the total strain $\varepsilon_{1, \mathrm{XRD}}$, determined from the diffraction data, and the macroscopic transverse strain measured at the edge of the sample, $\varepsilon_{1 \text {,vibro, }}$, is established first. Figure 6(a) shows that $\varepsilon_{1, \mathrm{XRD}}$ is larger than $\varepsilon_{1 \text {,vibro }}$ at every vibration velocity, where the blue squares denote the ratio between them. This difference can be explained by the internal mechanical strain and stress distribution and the different measurement positions on the sample. As demonstrated in the FEM simulation [Figs. 6(d)], strain and stress amplitudes in the 1 direction exhibit a sinusoidal distribution with a peak at the center of mass of the vibrating sample. This is in accordance with the theoretical distribution of a fundamental harmonic as opposed to a quasistatic electric field, which induces a homogeneous strain distribution. While $\varepsilon_{1 \text {,vibro }}$ is determined at the sample's edge (laser) and represents the average strain over the entire sample, $\varepsilon_{1, \mathrm{XRD}}$ is measured in the high-strain region in the sample's center (Fig. 1). The $\varepsilon_{1, \mathrm{XRD}} / \varepsilon_{1, \text { vibro }}$ ratio is in good agreement to the theoretical ratio of $\pi / 2(\sim 1.57)$ between the maximum and average values of a sinusoidal distribution. Note that the strain value calculated by FEM in the center of the sample, $\varepsilon_{1 \text {,FEM }}$

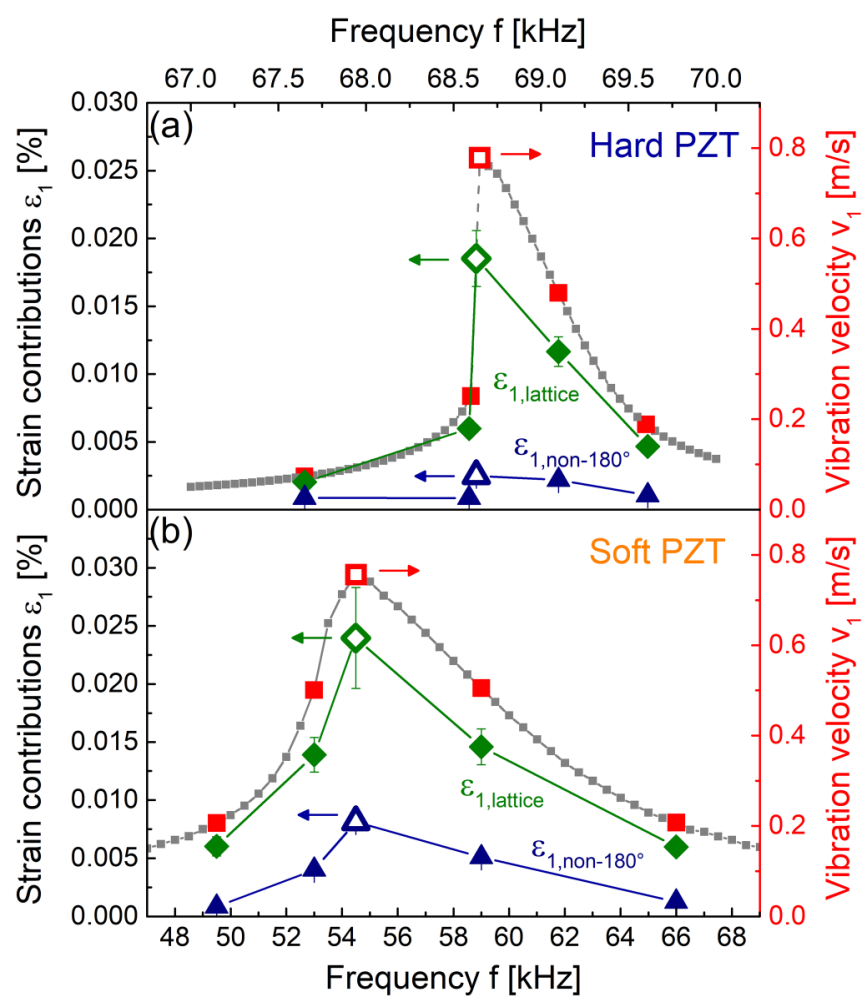

FIG. 5. Lattice strain $\left(\varepsilon_{1, \text { lattice }}\right)$ and strain contribution from non$180^{\circ}$ domain wall motion $\left(\varepsilon_{1, \text { non }-180^{\circ}}\right)$, determined from the XRD measurement, in comparison to the vibration velocity $\left(v_{1}\right)$, determined from pulse drive measurement, as a function of driving frequency $(f)$ measured at and near the resonance frequency $\left(f^{R}\right.$, marked by open symbols) for (a) hard and (b) soft PZT.

[star symbol in Fig. 6(a)], is in good agreement with the strain value established using diffraction.

It was demonstrated before that the main contribution to the overall sample strain comes from the lattice (intrinsic contribution), while the smaller domain wall contribution increases at large vibration velocity and is presumably the decisive reason for the $Q_{31}^{R}$ decrease (Figs. 3 and 4). However, the applied electric field is too low to explain the measured large strain.

Thus, we can conclude that in the case of resonance drive, the electric field is used only as a stimulus to bring the sample into resonant vibration and yields elastic energy through electromechanical coupling; however, the main contributor to the large $\varepsilon_{1 \text {,vibro }}$ is assumed to be the dynamic mechanical stress that builds up in a piezoceramic sample during resonance through the stored total elastic energy [22]. Besides producing a large $\varepsilon_{\text {lattice }}$, in perovskite ferroelectrics, this stress also drives the movement of ferroelastic non- $180^{\circ}$ domain walls and is thus the main driving force for obtaining large macroscopic strain.

These predictions are directly confirmed from Fig. 6(c) by evaluating individual contributions in Eq. (1). The vibration velocity-dependent piezoelectric coefficient $d_{31}$ and elastic compliance $s_{11}^{E}$ were determined by the pulse drive measurements [Fig. 6(b)]. Both coefficients increase at large vibration velocity indicating ferroelectric softening, as reported before [28]. The calculated $\varepsilon_{1, \mathrm{el}}$, determined from the applied electric 

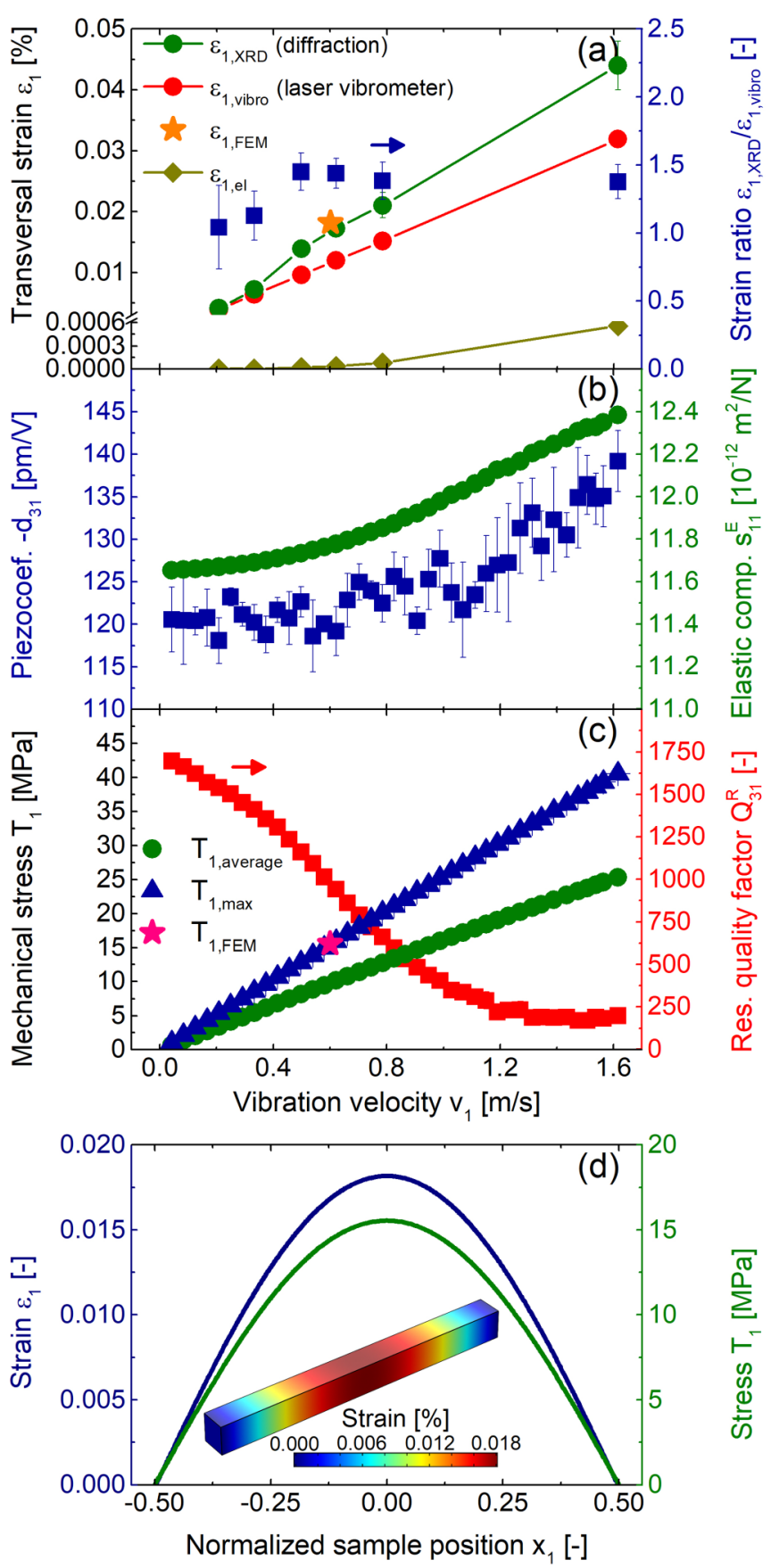

FIG. 6. (a) Transversal strain $\left(\varepsilon_{1}\right)$ versus the vibration velocity $\left(v_{1}\right)$ of hard PZT obtained from the diffraction measurement $\left(\varepsilon_{1, \mathrm{XRD}}\right.$, sample center) and macroscopic measurement with the laser vibrometer $\left(\varepsilon_{1, \text { vibro }}\right.$, sample edge), and calculated from the piezoelectric coefficient $\left(\varepsilon_{1, \text { el }}\right)$ and from the FEM model $\left(\varepsilon_{1, \text { FEM }}\right.$, sample center), as well as the strain ratio $\left(\varepsilon_{1, \mathrm{XRD}} / \varepsilon_{1, \mathrm{vibro}}\right)$. (b) Piezoelectric charge coefficient $\left(-d_{31}\right)$ and elastic compliance $\left(s_{11}^{E}\right)$ as a function of vibration velocity $\left(v_{1}\right)$ for hard PZT. (c) Resonance quality factor $\left(Q_{31}^{R}\right)$ determined from the pulse drive measurement, contrasted to the calculated average $\left(T_{1, \text { average }}\right)$ and maximum $\left(T_{1 \text { max }}\right)$ stress values in the sample and the FEM-simulated center strain value $\left(T_{1, \mathrm{FEM}}\right)$. (d) FEM sample model and traces of the transverse strain $\left(\varepsilon_{1}\right)$ and stress $\left(T_{1}\right)$ amplitude distributions in the 1 direction over the normalized sample position $\left(x_{1}\right)$ at $0.6 \mathrm{~m} / \mathrm{s}$ vibration velocity. field and the measured $d_{31}$, is about two orders of magnitude lower than the $\varepsilon_{1 \text {,vibro }}$ [Fig. 6(a)] and can be neglected, result-

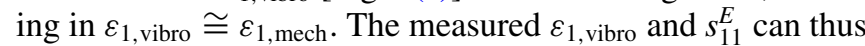
be used to calculate the average mechanical stress $T_{1 \text {,average }}$ [Fig. 6(c)]. Additionally, the stress amplitude in the sample's center, which we denote as maximum stress amplitude $T_{1, \max }$, can be calculated using the sample's density, $\rho$ [25]:

$$
T_{1, \max }=\sqrt{\frac{\rho}{s_{11}^{E}}} v_{1} .
$$

Both stress amplitudes increase at large vibration velocity, while the ratio $T_{1, \max } / T_{1 \text {,average }}$ is always close to $\frac{\pi}{2}$, which is the theoretical ratio of maximum and average values of a sinusoidal distribution. Additionally, the star in Fig. 6(c) marks the $T_{1, \max }$ determined from FEM calculations when the sample is vibrating at $0.6 \mathrm{~m} / \mathrm{s}$, which correlates well with the experimental value. These results confirm that the majority of the macroscopic strain in resonance originates from the mechanical contribution, enabled through the electromechanical coupling by the electric stimulus.

\section{CONCLUSIONS}

In conclusion, a direct structure-property correlation between increasing domain wall motion and decreasing $Q_{31}^{R}$ at high resonance vibration velocity was revealed. The domain wall motion is accompanied by internal loss, consequently, $Q_{31}^{R}$ values vary significantly, while other piezoelectric coefficients are reasonably constant over a wide range of vibration velocities. This provides direct structural evidence to confirm the long-standing hypothesis of the role of extrinsic contribution in the loss of piezoelectrics. Moreover, by quantifying the strain contributions for two PZT compositions, we demonstrate that the high-power stability of $Q_{31}^{R}$ is determined by the properties of the basic material and can be characterized by the attenuation parameter $\lambda$, while only small-signal $Q_{31}^{R}$ values can be altered by chemical modification. In contrast to off-resonance conditions, the applied electric field only triggers the vibration, while the emerging dynamic mechanical stress is the main driving force for the large vibration velocity and strain. The observed domain wall motion is thus the lattice's response to the stress by periodically switching the polar axis away from a direction of maximum compression and into a direction of maximum tension.

\section{ACKNOWLEDGMENTS}

This work was financially supported by the Deutsche Forschungsgemeinschaft under Grant No. 414073759 (KO 5100/3-1) and the Athene Young Investigator program of the TU Darmstadt. We acknowledge the European Synchrotron Radiation Facility (Exp. MA 3627) and Deutsches Elektronen-Synchrotron (DESY) (Hamburg, Germany; I20190080), a member of the Helmholtz Association HGF, for the provision of experimental facilities. Parts of this research were carried out at beamlines ID15 (ESRF) and P02.1 Deutsches Elektronen-Synchrotron (DESY) and we would like to thank Dr. Schökel for assistance. L.K.V. acknowledges and thanks the Alexander von Humboldt Foundation for financial support. 
[1] Q. M. Zhang, H. Wang, N. Kim, and L. E. Cross, Direct evaluation of domain-wall and intrinsic contributions to the dielectric and piezoelectric response and their temperature-dependence on lead-zirconate-titanate ceramics, J. Appl. Phys. 75, 454 (1994).

[2] T. J. Yang, V. Gopalan, P. J. Swart, and U. Mohideen, Direct Observation of Pinning and Bowing of a Single Ferroelectric Domain Wall, Phys. Rev. Lett. 82, 4106 (1999).

[3] D. Damjanovic and M. Demartin, Contribution of the irreversible displacement of domain walls to the piezoelectric effect in barium titanate and lead zirconate titanate ceramics, J. Phys.: Condens. Matter 9, 4943 (1997).

[4] D. Damjanovic, Contributions to the piezoelectric effect in ferroelectric single crystals and ceramics, J. Am. Ceram. Soc. 88, 2663 (2005).

[5] H. J. Lee, T. Shimizu, H. Funakubo, Y. Imai, O. Sakata, S. H. Hwang, T. Y. Kim, C. Yoon, C. Dai, L. Q. Chen, S. Y. Lee, and J. Y. Jo, Electric-Field-Driven Nanosecond Ferroelastic-Domain Switching Dynamics in Epitaxial Pb(Zr,Ti)O3 Film, Phys. Rev. Lett. 123, 217601 (2019).

[6] T. N. Yang, B. Wang, J. M. Hu, and L. Q. Chen, Domain Dynamics under Ultrafast Electric-Field Pulses, Phys. Rev. Lett. 124, 107601 (2020).

[7] D. A. Hall, Nonlinearity in piezoelectric ceramics, J. Mater. Sci. 36, 4575 (2001).

[8] A. Pramanick, D. Damjanovic, J. E. Daniels, J. C. Nino, and J. L. Jones, Origins of electro-mechanical coupling in polycrystalline ferroelectrics during subcoercive electrical loading, J. Am. Ceram. Soc. 94, 293 (2011).

[9] M. J. Hoffmann, M. Hammer, A. Endriss, and D. C. Lupascu, Correlation between microstructure, strain behavior, and acoustic emission of soft PZT ceramics, Acta Mater. 49, 1301 (2001).

[10] J. T. Reszat, A. E. Glazounov, and M. J. Hoffmann, Analysis of intrinsic lattice deformation in PZT-ceramics of different compositions, J. Eur. Ceram. Soc. 21, 1349 (2001).

[11] K. S. Lee, Y. K. Kim, S. Baik, J. Kim, and I. S. Jung, In situ observation of ferroelectric 90 degrees-domain switching in epitaxial $\mathrm{Pb}(\mathrm{Zr}, \mathrm{Ti}) \mathrm{O}_{3}$ thin films by synchrotron $\mathrm{x}$-ray diffraction, Appl. Phys. Lett. 79, 2444 (2001).

[12] J. L. Jones, M. Hoffman, J. E. Daniels, and A. J. Studer, Direct measurement of the domain switching contribution to the dynamic piezoelectric response in ferroelectric ceramics, Appl. Phys. Lett. 89, 092901 (2006).

[13] J. E. Daniels, T. R. Finlayson, A. J. Studer, M. Hoffman, and J. L. Jones, Time-resolved diffraction measurements of electricfield-induced strain in tetragonal lead zirconate titanate, J. Appl. Phys. 101, 094104 (2007).

[14] J. L. Jones, A. Pramanick, J. C. Nino, S. M. Motahari, E. Ustundag, M. R. Daymond, and E. C. Oliver, Time-resolved and orientation-dependent electric-field-induced strains in lead zirconate titanate ceramics, Appl. Phys. Lett. 90, 172909 (2007).

[15] J. E. Daniels, A. Pramanick, and J. L. Jones, Time-resolved characterization of ferroelectrics using high-energy X-ray diffraction, IEEE Trans. Ultrason. Ferr. 56, 1539 (2009).

[16] K. Nakamura, in Ultrasonic Transducers: Materials and Design for Sensors, Actuators and Medical Applications (Woodhead, Cambridge, UK, 2012), Vol. 1, pp. 264-276.

[17] K. Uchino, in Advanced Piezoelectric Materials (Woodhead, Cambridge, UK, 2017), Vol. 1, pp. 647-754.
[18] Standards EN 50324-3, Piezoelectric Properties of Ceramic Materials and Components Part 3: Methods of Measurement-High Power (European Committee for Electrotechnical Standardization, Brussels, Belgium, 2002).

[19] K. Uchino and S. Hirose, Loss mechanisms in piezoelectrics: How to measure different losses separately, IEEE Trans. Ultrason. Ferr. 48, 307 (2001).

[20] S. Takahashi, Y. Sasaki, and S. Hirose, Driving electric field effects on piezoelectric transducers, Jpn. J. Appl. Phys. 36, 3010 (1997).

[21] S. Takahashi and S. Hirose, Vibration-level characteristics of lead-zirconate-titanate ceramics, Jpn. J. Appl. Phys. 31, 3055 (1992).

[22] R. Gerson, Dependence of mechanical-Q and youngs modulus of ferroelectric ceramics on stress amplitude, J. Acoust. Soc. Am. 32, 1297 (1960).

[23] H. Shekhani, T. Scholehwar, E. Hennig, and K. Uchino, Characterization of piezoelectric ceramics using the burst/transient method with resonance and antiresonance analysis, J. Am. Ceram. Soc. 100, 998 (2017).

[24] J. Y. Jo, S. M. Yang, T. H. Kim, H. N. Lee, J. G. Yoon, S. Park, Y. Jo, M. H. Jung, and T. W. Noh, Nonlinear Dynamics of Domain-Wall Propagation in Epitaxial Ferroelectric Thin Films, Phys. Rev. Lett. 102, 045701 (2009).

[25] M. Umeda, S. Takahashi, Y. Sasaki, K. Nakamura, and S. Ueha, Vibration stress and temperature dependence of piezoelectric resonators with lead-zirconate-titanate ceramics, Electron. Commun. Jpn. 83, 1 (2000).

[26] Standards EN 50324-1, Piezoelectric Properties of Ceramic Materials and Components Part 1: Terms and Definitions (European Committee for Electrotechnical Standardization, Brussels, Belgium, 2002).

[27] M. Umeda, K. Nakamura, and S. Ueha, The measurement of high-power characteristics for a piezoelectric transducer based on the electrical transient response, Jpn. J. Appl. Phys. 37, 5322 (1998).

[28] M. Slabki, J. Wu, M. Weber, P. Breckner, D. Isaia, K. Nakamura, and J. Koruza, Anisotropy of the high-power piezoelectric properties of $\mathrm{Pb}(\mathrm{Zr}, \mathrm{Ti}) \mathrm{O}_{3}$, J. Am. Ceram. Soc. 102, 6008 (2019).

[29] See Supplemental Material at http://link.aps.org/supplemental/ 10.1103/PhysRevB.103.174113 for details on the diffraction data based strain analysis, the experimental and fitting parameters, and the determined strain contributions.

[30] G. B. Vaughan, R. Baker, R. Barret, J. Bonnefoy, T. Buslaps, S. Checchia, D. Duran, F. Fihman, P. Got, and J. Kieffer, ID15A at the ESRF-a beamline for high speed operando X-ray diffraction, diffraction tomography and total scattering, J. Synchrotron Radiat. 27, 515 (2020).

[31] E. Zolotoyabko, J. P. Quintana, B. H. Hoerman, and B. W. Wessels, Fast time-resolved X-ray diffraction in BaTiO3 films subjected to a strong high-frequency electric field, Appl. Phys. Lett. 80, 3159 (2002).

[32] A. P. Hammersley, S. O. Svensson, M. Hanfland, A. N. Fitch, and D. Hausermann, Two-dimensional detector software: From real detector to idealised image or two-theta scan, High Pressure Res. 14, 235 (1996).

[33] J. L. Jones, E. B. Slamovich, and K. J. Bowman, Domain texture distributions in tetragonal lead zirconate titanate by X-ray and neutron diffraction, J. Appl. Phys. 97, 034113 (2005). 
[34] M. R. Daymond, The determination of a continuum mechanics equivalent elastic strain from the analysis of multiple diffraction peaks, J. Appl. Phys. 96, 4263 (2004).

[35] J. L. Jones, M. Hoffman, and K. J. Bowman, Saturated domain switching textures and strains in ferroelastic ceramics, J. Appl. Phys. 98, 024115 (2005).

[36] V. S. Postnikov, V. S. Pavlov, and S. K. Turkov, Internal friction in ferroelectrics due to interaction of domain boundaries and point defects, J. Phys. Chem. Solids 31, 1785 (1970).

[37] K. Carl and K. H. Hardtl, Electrical aftereffects in $\mathrm{Pb}(\mathrm{Ti}, \mathrm{Zr}) \mathrm{O} 3$ ceramics, Ferroelectrics 17, 473 (1978).

[38] U. Robels and G. Arlt, Domain-wall clamping in ferroelectrics by orientation of defects, J. Appl. Phys. 73, 3454 (1993).

[39] S. Takahashi, S. Hirose, and K. Uchino, Stability of PZT piezoelectric ceramics under vibration level change, J. Am. Ceram. Soc. 77, 2429 (1994).

[40] S. Hirose, M. Aoyagi, Y. Tomikawa, S. Takahashi, and K. Uchino, High power characteristics at antiresonance frequency of piezoelectric transducers, Ultrasonics 34, 213 (1996).

[41] J. F. Nye, in Physical Properties of Crystals: Their Representation by Tensors and Matrices (Oxford University Press, New York, 1985), Vol. 1, pp. 93-105.

[42] D. Damjanovic, M. Demartin, H. S. Shulman, M. Testorf, and N. Setter, Instabilities in the piezoelectric properties of ferroelectric ceramics, Sens. Actuators, A 53, 353 (1996).

[43] J. L. Jones, E. Aksel, G. Tutuncu, T. M. Usher, J. Chen, X. R. Xing, and A. J. Studer, Domain wall and interphase boundary motion in a two-phase morphotropic phase boundary ferroelectric: Frequency dispersion and contribution to piezoelectric and dielectric properties, Phys. Rev. B 86, 024104 (2012).

[44] K. Uchino, J. H. Zheng, A. Joshi, Y. H. Chen, S. Yoshikawa, S. Hirose, S. Takahashi, and J. W. C. De Vries, High power characterization of piezoelectric materials, J. Electroceram. 2, 33 (1998).

[45] Y. Doshida, H. Shimizu, Y. Mizuno, and H. Tamura, High-power properties and miniature ultrasonic motor of $(\mathrm{Sr}, \mathrm{Ca})_{2} \mathrm{Na}_{5} \mathrm{NbO}_{15}$ piezoelectric ceramics, Ceram. Int. 39, S721 (2013).

[46] M. Hejazi, E. Taghaddos, E. Gurdal, K. Uchino, and A. Safari, High power performance of manganese-doped BNT-based Pbfree piezoelectric ceramics, J. Am. Ceram. Soc. 97, 3192 (2014).

[47] D. Damjanovic and M. Demartin, The Rayleigh law in piezoelectric ceramics, J. Phys. D: Appl. Phys. 29, 2057 (1996).

[48] D. A. Hall and P. J. Stevenson, High field dielectric behaviour of ferroelectric ceramics, Ferroelectrics 228, 139 (1999).

[49] H. Beige and G. Schmidt, Electro-mechanical resonances for investigating linear and non-linear properties of dielectrics, Ferroelectrics 41, 39 (1982).

[50] S. Priya, D. Viehland, A. V. Carazo, J. Ryu, and K. Uchino, High-power resonant measurements of piezoelectric materials: Importance of elastic nonlinearities, J. Appl. Phys. 90, 1469 (2001). 